\title{
REVIEW
}

\section{Alternative haematotoxicological testing}

\section{Josef Berger}

Faculty of Health and Social Studies, University of South Bohemia in České Budějovice, Czech Republic

Received $16^{\text {th }}$ October 2009.

Revised $22^{\text {nd }}$ November 2009.

Published online $5^{\text {th }}$ January 2010.

\begin{abstract}
Summary
Standard haematological procedures in preclinical subchronic and chronic toxicity studies are carried out on dogs, rats and other mammals. In vitro clonic assays CFU-GM, BFU-E, CFU-E, CFU-Mgkr and pluripotent stem cells are used in screening candidate compounds to predict acute cytopenias, but they are not able to reveal the risk of suppression which can develop after repeated administration. No in silico studies have yet been published in haematotoxicology. More recent haematotoxicological in vivo models represent invertebrates: they can be used in both ecotoxicology and in the screening of myelotoxicity.
\end{abstract}

Key words: myelotoxicity; alternative testing; in vitro; in silico; invetebrate

\section{INTRODUCTION}

Haematological studies were among the first to be incorporated into routine toxicology. Classical preclinical haematoxicological examinations, which detect the adverse effects of various xenobiotics, are carried out on laboratory animals in subchronic and chronic organismal studies (Berger 1987). The use of laboratory animal models is limited by restrictions driven by social and economical concerns (cf. Knight 2008).

Alternatives to mammalian models can be in vitro assays, in silico analyses or the use of invertebrates.
In vitro clonogenic assays have been established for forty years; in toxicology for the last twenty years. Computerised methods have also been evaluated for several decades, but primarily for the prediction of the desired effects of newly developed drugs. A goal in predictive toxicology is the use of in vitro methods and database development to make toxicological predictions (Guengerich and MacDonald 2007).

Invertebrate models are necessary in many areas of biomedical research but it seems that they go often unheeded in ecotoxicology (Berger 2009a).

The aim of our contribution is shortly to discuss the main problems current in haematoxicology.

\section{NON-ANIMAL ASSAYS}

Cytotoxic drugs can be also evaluated by in vitro clonogenic assays to investigate changes in the proliferation and differentiation of stem cells (Pessina et al. 2005, for review). The prediction of in vivo 
acute neutropenia using a CFU-GM (colony forming unit-granulocyte-macrophage) assay seems to be the most valuable (Masubuchi 2006). Pluripotent haematopoietic stem cell, CFU-Mgkr (megakaryocyte) and BFU-E or CFU-E (erythroid) assays are also useful. They can predict acute thrombocytopenia, anaemia and aplastic anaemia.

On the other hand, colony growth inhibition granulocyte-macrophage colony-forming-units (GM-CFC) may not be correlated with the degree of myelotoxicity in the same patient (Sessa et al. 1999). Comparing the differential effects of antitumour drugs on mature haematopoietic stem cells such as GM-CFC and undifferentiated long-term culture-initiating cells, haematopoietic progenitors in vitro may not represent a valid model for the pattern of myelotoxicity observed in clinical studies carried on human subjects (Ghielmini et al. 1999). Haematological in vitro tests, moreover, seem not to be able to reveal the risk of the suppression which can develop after repeated administration of an evaluated compound.

In silico analysis is asuming some importance in toxicology (Knight 2008, Nigsch et al. 2009), especially for predicting preclinical toxicological endpoints, clinical adverse effects, and the metabolism of pharmaceutical substances (Valerio 2009). A more recent summary of in silico systems for toxicity predictions is available in the literature (Muster et al. 2008). Although included in haematology for the last ten years, this approach is limited to the analysis of genes and gene expression related to malignancy (Stegmaier 2009). Toxicological database development also covers haematological data but no in silico studies have yet been published in haematotoxicology.

\section{NON-MAMMALIAN BIOMODELS}

Invertebrates are phylogenetically distant from humans and their use for the prediction of the side-effects of various compounds in human subjects can therefore be disputed. On the other hand, we have known for many years that the Ames test on microorganisms is a very useful screening of mutagenicity. Moreover, systematic reviews of toxicological results demonstrate that mammalian models have not been substantially consistent with clinical outcomes in many studies (Knight 2007).

Invertebrates are already used in several areas of biomedical research, for example, ageing research (Flatt and Schmidt 2009), genetics and neuroscience (Lu 2009, Spokony and Restifo 2009, Yang and Lu
2009), paediatrics and physiology (Zhou et al. 2009), and oncology (Vaccari and Bilder 2009). There are also several studies which used invertebrates in toxicological research (e.g., Berger et al. 2003, Cummings and Kavlock 2005, Park and Lee 2007, Verhofstad et al. 2008, Choudhuri 2009).

Xenobiotics influence the total number of circulating haemocytes; they usually decrease it and change differentials (e.g., Berger et al. 2003, Auffret et al. 2004, Yildiz and Benli 2004, Szczerbina et al. 2008, Oweson and Hernroth 2009). A very important immune function of haemocytes as well as human neutrophils and monocytes is phagocytosis (Bouchard et al. 1999, Auffret et al. 2004, Cammarata et al. 2007, Russo et al. 2007, De Guise et al. 2008, Gagné et al. 2008, Oweson et al. 2008). Alterations in phagocytosis are a consequence of changes in the cytoskeleton (Cima et al. 2008, Menin et al. 2008).

Some xenobiotics induce programmed cell death, which can be diagnosed also in haemocytes (Cima et al. 2008, De Guise et al. 2008). A similar situation concerns the detection of haemocyte necrosis (Sung et al. 2003, De Guise et al. 2008).

Haemocytes divide in haemolymph. Their mitotic index (Oweson 2008) has a similar diagnostic role as the percentage of mitosis in the bone marrow of mammals.

Haemocytes make it possible to detect genotoxic injuries by the micronucleus test (Dopp et al. 1996, Carvalho Pinto-Silva et al. 2005, Binelli et al. 2008).

\section{CURRENT TRENDS}

The main area where invertebrates are used for haematotoxicology, seems to be ecotoxicology because contaminants can adversely affect immune functions in both human subjects and wildlife (Galloway and Depledge 2001).

The morphology of invertebrate haemocytes is similar to the mammalian leucocyte (Berger 2009a). Haemocytes (or coelomocytes) may synthesize immune proteins and agglutinins and phagocytise foreign particles (Lavine and Strand 2002). Although invertebrates have no erythrocytes, haemolytic potential can be tested by detecting changes in haemocyte morphology (Berger 2009b).

Invertebrates can be used as in vivo models for haematotoxicological screening of newly developed drugs or chemicals in occupational environments. To reach a better prediction of adverse effects, further haematology research of invertebrate species and their haematokinetics seems to be desirable. 


\section{REFERENCES}

Auffret M, Duchemin M, Rousseau S, Boutet I, Tanguy A, Moraga D, Marhic A: Monitoring of immunotoxic responses in oysters reared in areas contamined by the "Erika" oil spill. Aquat Living Resour 17:297-302, 2004.

Berger J: Haematological preclinical recording of adverse drug reactions - current status, problems, and needs. Part II. Diagnostic aspects. Folia Haematol 114:16-41, 1987.

Berger J: Preclinical testing on insects predicts human haematotoxic potentials. Lab Anim 43:328-332, 2009a.

Berger J: New haemolytic potential assay on an alternative insect model. Basic Clin Pharmacol Toxicol 105:315-318, 2009b.

Berger J, Walczysko S, Pávková J, Gutzeit HO: Effects of genistein on insect haemocytes. J Appl Biomed 1:161-168, 2003.

Binelli A, Riva C, Cogni D, Provini A: Genotoxic effects of p,p'-DDT (1,1,1 trichloro2,2-bis-[chlorophenyl] ethane) and its metabolites in Zebra mussel (D. polymorpha) by SCGE assay and micronucleus test. Environ Mol Mutagen 49:406-415, 2008.

Bouchard N, Pelletier É, Fournier M: Effects of butyltin compounds on phagocytic activity of hemocytes from three marine bivalves. Environ Toxicol Chem 18:519-522, 1999.

Cammarata M, Parisi MG, Benenati G, Arizza V, Cillari T, Piazzese D, Gianguzza A, Vazzana M, Vizzini A, Parrinello $\mathrm{N}$ : In vitro effects of methylmercury on ascidian (Styela plicata) immunocyte responses. Appl Organomet Chem 21:1022-1028, 2007.

Carvalho Pinto-Silva C, Creppy EE, Matias WG: Micronucleus test in mussels Perna perna fed with the toxic diniflagelate Prorocentrum lima. Arch Toxicol 79:422-426, 2005.

Choudhuri S: Looking back to the future: From the development of the gene concept to toxicogenomics. Toxicol Mech Methods 19:263-277, 2009.

Cima F, Bragadin M, Ballarin L: Toxic effects of new antifouling compounds on tunicate haemocytes. I. Sea-Nine $211^{\mathrm{TM}}$ and chlorothalonil. Aquat Toxicol 86:299-312, 2008.

Cummings A, Kavlock K: A systems biology approach to developmental toxicology. Reprod Toxicol 19:281-290, 2005.

De Guise S, Goedken M, Morsey B, Maratea J, Sidor I, Perkins C: Development of new methods to ossess invertebrate immunology and immunotoxicology in aquaculture: Oysters and lobsters as examples. Int $\mathrm{J}$ Environ Pollut 33:365-380, 2008.

Dopp E, Barker CM, Schiffman D, Reinisch CL: Detection of micronuclei in hemocytes of Mya arenaria association with leukemia and induction with an alkylating agent. Aquat Toxicol 34:31-45, 1996.

Flatt T, Schmidt PS: Integrating evolutionary and molecular genetics of aging. Biochim Biophys Acta 1790:951-962, 2009.

Gagné F, Auclair J, Turcotte P, Fournier M, Gagnon C, Sauvé S, Blaise C: Ecotoxicity of CdTe quantum dots to freshwater mussels: Impact on immune system, oxidative stress and genotoxicity. Aquat Toxicol 86:333-340, 2008.

Galloway TS, Depledge MH: Immunotoxicity in invertebrates: Measurement and ecotoxicological relevance. Ecotoxicology 10:5-23, 2001.

Ghielmini M, Bosshard G, Pampallona S, Genini D, Bernier J, Sessa C: Differential toxicity of anticancer drugs on late (GM-CFC) and early (LTC-IC) hemopoetic progenitors in vitro. Cell Biol Toxicol 15:395-404, 1999.

Guengerich FP, MacDonald JS: Applying mechanisms of chemical toxicity to predict drug safety. Chem Res Toxicol 20:344-369, 2007.

Knight A: Animal experiments scrutinised: Systematic reviews demonstrate poor human clinical and toxicological utility. ALTEX 24:320-325, 2007.

Knight A: Non-animal methodologies within biomedical research and toxicity testing. ALTEX 25:213-231, 2008.

Lavine MD, Strand MR: Insect hemocytes and their role in immunity. Insect Biochem Mol Biol 32:1295-1309, 2002.

$\mathrm{Lu}$ B: Recent advances in using Drosophila to model neurodegenerative diseases. Apoptosis 14:1008-1020, 2009.

Masubuchi N: Risk assessment of human myelotoxicity of anticancer drugs: A predictive model and the in vitro colony forming unit granulocyte/macrophage (CFU-GM) assay. Pharmazie 61:135-139, 2006.

Menin A, Ballarin L, Bragadin M, Cima F: Immunotoxicity in ascidians: Antifouling compounds alternative to organotins - II. The case of Diuron and TCMS pyridine. J Environ Sci Health B 43:644-654, 2008.

Muster W, Breidenbach A, Fischer H, Kirchner S, Miller L, Pähler A: Computational toxicology in drug development. Drug Discov Today 13:303-310, 2008 .

Nigsch F, Macaluso NJM, Mitchell JBO, Zmuidinavicius D: Computational toxicology: 
An overview of the sources of data and of modelling methods. Expert Opin Drug Metab Toxicol 5:1-14, 2009.

Oweson C, Hernroth B: A comparative study on the influence of manganese on the bactericidal response of marine invertebrates. Fish Shellfish Immunol 27:500-507, 2009.

Oweson C, Sköld H, Pinsino A, Matranga V, Hernroth B: Manganese effects on haemopoietic cells and circulating coelomocytes of Asterias rubens (Linnaeus). Aquat Toxicol 89:75-81, 2008.

Park BS, Lee SE: Proteomics in insecticide toxicology. Moll Cell Toxicol 3:11-18, 2007.

Pessina A, Malerba I, Gribaldo L: Hematotoxicity testing by cell clonogenic assay in drug development and preclinical trials. Curr Pharm Des 11:1055-1065, 2005.

Russo J, Lefeuvre-Orfila L, Lagadic L: Hemocyte-specific response to the peroxidizing herbicide fomesafen in the pond snail Lymnaea stagnalis (Gastropoda, Pulmonata). Environ Pollut 146:420-427, 2007.

Sessa C, Zucchetti M, Ghielmini M, Bauer J, D'Incalci M, de Jong J, Naegele H, Rossi S, Pacciarini MA, Domenigoni L, Cavalli F: Phase I clinical and pharmacological study of oral methoxymorpholinyl doxorubicin (PNU 152243). Cancer Chemother Pharmacol 44:403-410, 1999.

Spokony RF, Restifo LL: Broad complex isoforms have unique distributions during central nervous system metamorphosis in Drosophila melanogaster. J Comp Neurol 517:15-36, 2009.

Stegmaier K: Genomic approaches to small molecule discovery. Leukemia 23:1226-1235, 2009.
Sung H-H, Kao W-Y, Su Y-J: Effects and toxicity of phthalate esters to hemocytes of giant freshwater prawn, Macrobrachium rosenbergii. Aquat Toxicol 64:25-37, 2003.

Szczerbina T, Banach Z, Tylko G, Pyza E: Toxic effects of acrylamide on survival, development and haemocytes of Musca domestica. Food Chem Toxicol 46:2316-2319, 2008.

Vaccari T, Bilder D: At the crossroads of polarity, proliferation and apoptosis: The use of Drosophila to unravel the multifaceted role of endocytosis in tumor suppression. Mol Oncol 3:354-365, 2009.

Valerio LG, Jr.: In silico toxicology for the pharmaceutical science. Toxicol Appl Pharmacol 241:356-370, 2009.

Verhofstad N, Linschooten JO, van Benthem J, Dubrova YE, van Steeg H, van Schooten FJ, Godschalk RL: New methods for assessing male germ line mutations in humans and genetic risks in their offspring. Mutagenesis 23:241-247, 2008.

Yang Y, Lu B: Mitochondrial morphogenesis, distribution, and Parkinson disease: Insights From PINK1. J Neuropathol Exp Neurol 68:953-963, 2009.

Yildiz HY, Benli ACK: Nitrite toxicity to crayfish, Astacus leptodactylus, the effects of sublethal nitrite exposure on hemolymph nitrite, total hemocyte counts, and hemolymph glucose. Ecotoxicol Environ Saf 59:370-375, 2004.

Zhou D, Visk DW, Haddad GG: Drosophila, a golden bug, for the dissection of the genetic basis of tolerance and susceptibility to hypoxia. Pediatr Res 66:239-247, 2009. 